\title{
Novel-integrated process for production of bio-organic fertilizer via swine manure composting
}

\author{
Yan Chen ${ }^{1}$, Xiaoyu $\mathrm{Li}^{1,2}$, Shuying $\mathrm{Li}^{3}$, Yongping $\mathrm{Xu}^{1,2,3^{\dagger}}$ \\ ${ }^{1}$ School of Bioengineering, Dalian University of Technology, Dalian 116024, PR China \\ ${ }^{2}$ Ministry of Education Center for Food Safety of Animal Origin, Dalian 116620, PR China \\ ${ }^{3}$ Dalian SEM Bio-Engineering Technology Co., Ltd. Dalian 116620, PR China
}

\begin{abstract}
The traditional method of producing bio-organic fertilizer contains two consecutive composting stages which, however, is time-consuming and requires high input of energy and cost. This study attempted to combine two consecutive stages into one single step. Paenibacillus polymyxa, as an antagonistic strain to control anthracnose in Chinese Pakchoi, was used as inoculum to obtain bio-organic fertilizers through two approaches: two consecutive stages (TCS) composting as control and the proposed one step through (OST) composting. The resulting two bio-organic fertilizers produced by TCS and OST were tested for the disease incidence and disease resistant index, and the fertility on growth of Chinese Pakchoi. The results of relative abundance of P. polymyxa showed no significant difference between two kinds of bio-organic fertilizers in phylum, family and genus. The disease incidence of bio-organic fertilizers produced by OST and TCS reduced by $61.40 \%$ and $68.42 \%$, respectively, as compared to the control group, where the difference was not significant. The growth promoting effect was remarkable, although the difference in the fertility on the growth was not significant. Compared with the traditional TCS method, the novel OST method could improve efficiency of bio-organic fertilizer production by $42.86 \%$ per year according to this study.
\end{abstract}

Keywords: A novel method, Bio-organic fertilizer, Compost, Disease incidence, Production efficiency

\section{Introduction}

Control of plant disease using chemical pesticides is one of the most common strategies around the world [1]. However, it is not environmental-friendly to plant soil and human beings due to the toxicity of pesticides. Moreover, the chemical pesticides used have negative effects on some beneficial microbes that facilitate soil nutrient recycle and nutrient uptake by plant roots [2]. With the increasing concern to food safety and environment changes, biological control methods have become increasingly used as an alternative to chemical pesticides and been considered as a promising strategy for plant disease management [3]. Bio-organic fertilizer has become one of the main methods of biological control due to its special function of disease-resistant strains [4, 5], which is main coming from composting technology. A large number of solid wastes, such as crop straw, livestock and poultry manure, have been produced from agricultural industry and animal husbandry. China accounts for more than $50 \%$ of the total pig production in the world, resulting in production of an estimated 618 million tons of manure every year [6]. Considering the quantity and disposal of swine manure, composting technology has been gradually and widely adopted worldwide due to its effectiveness in valorization and pollutant reduction of the swine manure [7]. Besides, the end products of the compost are organic fertilizer or bio-organic fertilizer which are harmless to soil biota and plants, considering as a sustainable strategy for wasting resource utilization $[8,9]$. Therefore, the production and use of bio-organic fertilizer in arable land could not only realize the harmlessness and resourcefulness of livestock and poultry manure, but also alleviate heavy use of chemical pesticides and insecticides, thus saving investment cost for farmers and reducing potential soil and water pollution.

Bio-organic fertilizer has obvious advantages for environment as well as for health of human beings. Many researchers have studied and developed methods of producing bio-organic fertilizer in their related fields [3, 9]. One of the traditional or widely used methods of producing bio-organic fertilizer [10] is the composting
This is an Open Access article distributed under the terms of the Creative Commons Attribution Non-Commercial License (http://creativecommons.org/licenses/by-nc/3.0/) which permits unrestricted non-commercial use, distribution, and reproduction in any medium, provided the original work is properly cited.

Copyright (C) 2021 Korean Society of Environmental Engineers
Received December 26, 2019 Accepted April 01, 2020

${ }^{\dagger}$ Corresponding author

Email: xyp560104@hotmail.com

Tel: +86-411-8470-9800 Fax: 86-411-8470-9800

ORCID: 0000-0003-3891-9015 
process containing two consecutive stages (TCS) of composting fermentation. In the first stage, the livestock and poultry manure was fermented via aerobic composting technology to the phase of maturity followed by the second stage starting from inoculation of microbial agents with ability to control plant diseases. The second stage was subjected to the same phases of mesophilic, thermophilic and cooling or maturity as in the first stage before completion of the whole process [11]. In general, besides normal monitoring and management for each stage of composting during the process of traditional bio-organic fertilizer production, it is necessary to control the homogeneity of the composting pile after microbial agents inoculation through periodically turning and stirring [12]. Two consecutive stages of composting fermentation with management and monitoring throughout the whole process require extra workforce, utilities, processing period, cost investment, and lowering the overall productivity of the composting process.

In this study, we combined the two consecutive stages of composting used for traditional production of bio-organic fertilizer into one step through (OST) composting in the lab scale. This new method of producing bio-organic fertilizer was proposed as the following steps: inoculating microorganisms with the ability of disease control into the piles at the beginning of composting. And then, the fermentation of composting followed by single-continuous composting subjecting to phases of mesophilic, thermophilic, cooling and maturity. When composting finished, the end product of the new OST composting, bio-organic fertilizer, contained a certain amount of biological control microbes which could meet the standard of microbial organic fertilizers of China (NY 884-2012). Moreover, the bio-organic fertilizer from the new OST method could have the same diseases incidence and fertility on the growth of crops as the TCS method, whereas reducing processing time period, thus production and investment cost and extra workforce.

Paenibacillus polymyxa belonged to Bacillus spp. and was used as an antagonistic strain with the ability to control the plant disease by disturbing the growth of pathogen of Chinese Pakchoi anthracnose $[13,14]$. It is one of the most popular vegetable diseases and seriously affects the plant yield. Microbial agents contained $P$. polymyxa was used as inoculum to produce bio-organic fertilizers via traditional TCS method and the proposed OST method. The main objective of this study was to evaluate the feasibility of using novel OST method for production of bio-organic fertilizer and the subsequent diseases incidence and fertility on the growth of Chinese Pakchoi in pot experiment by applying different loadings of the bio-organic fertilizers produced by the methods of OST and TSC.

\section{Material and Methods}

\subsection{Agent Preparation}

The microorganisms used as biological control agents in this study were two strains of $P$. polymyxa-ACCC 03043 and $P$. polymyxa-ACCC 10369, which were obtained from Agricultural Culture Collection of China (ACCC). The inocula of both strains were evenly mixed at the ratio of $1: 1(\mathrm{v} / \mathrm{v})$ before inoculating into the composting piles. Colletotrichum higginsianum -ACCC 37053 obtained from ACCC was used as disease pathogen to infect Chinese Pakchoi in pot experiment for evaluating the disease incidence and disease resistant index of the bio-organic fertilizers produced by OST and TSC methods. The inoculum of biological control agents and infection agents were prepared as follows: I) biological control agents, P. polymyxa -ACCC 03043 and P. polymyxa -ACCC 10369 were cultured in Lysogeny Broth (LB) with $1000 \mathrm{~mL}$ suspension of $1 \times 10^{9}$ colony forming units (CFUs); II) infection agents, $C$. higginsianum was cultured in Potato Dextrose Agar (PDA) plate at $28^{\circ} \mathrm{C}$ for 5 to $7 \mathrm{~d}$, followed by culturing with $1,000 \mathrm{~mL}$ suspension of $1 \times 10^{6} \mathrm{CFUs}$.

\subsection{Composting Design}

The composting experiments were conducted in polyethylene bins each with the dimension of $75 \times 55 \times 45 \mathrm{~cm}$ (length $\times$ width $\times$ height). The outer layer of the composting box was wrapped with foam having thickness of $2.5 \mathrm{~cm}$. The box cover had five evenly distributed openings $(2.5 \times 2.5 \mathrm{~cm})$ at four corners and center for ventilation. Two ventilating pipes each with a diameter of $5 \mathrm{~cm}$ were located at the bottom of the composting box. Five polyethylene fiber rods were evenly distributed to five locations of the composting pile (four corners and center). Each polyethylene fiber rod was equipped with three thermocouples (TES-TP-K01, China), which were located at three depths of $10 \mathrm{~cm}, 20 \mathrm{~cm}$ and $30 \mathrm{~cm}$ from the bottom of the box. The ambient temperature and temperatures measured by each thermocouple in the box were recorded twice per day (9:00 am and 5:00 pm) by digital thermometer receiver (TES-1310, China), and then, calculated the average value of temperature.

The swine manure used in this study was collected from the Xinlei intensive pig farming facility in Dalian, China, and had $\mathrm{pH}$ of 7.87 , moisture content of $69.27 \%$, total organic carbon content of $33.08 \%$, total nitrogen content of $2.34 \%$, and the carbon to nitrogen $(\mathrm{C} / \mathrm{N})$ ratio of 14.17 . The swine manure and corn straw (0.5 - 1 cm lengths) were well mixed and then loosely loaded into each experimental box at loading rate of about $110 \mathrm{~kg}$. The $\mathrm{C} / \mathrm{N}$ ratio and moisture content of composting mixture were adjusted to about 25:1 and 65\%, respectively. The design of composting experiments was showed in Table 1. For the reason that the method of producing bio-organic fertilizer suffered two different kinds of composting fermentation, CK-1 was used as control group for OST and CK-2 was used as control group for TCS. Three replications were performed for each treatment. The compost piles were turned for proper mixing on day $5,10,20$, and 30 . The total single composting time (including phases of mesophilic, thermophilic, cooling and maturity) was 35 days. The biological control agents were inoculated into the composting piles at $5 \%(\mathrm{v} / \mathrm{w})$ at the initial phase of composting using OST method, while at the maturation phase of composting using TCS method. When the temperatures of all the composting piles were close to the ambient temperature and stabilized, the composting process was stopped in group CK-1 and group OST with collection of final samples. However, the composting of group TCS and CK-2 continued with the second stage of composting fermentation in TCS starting after inoculation with biological control agents. The second stage composting for both groups CK-2 and TCS lasted for another 14 days until finish. Three parallel samples (about $1 \mathrm{~kg}$ for each) were collected by mixing subsamples from the upper, central, and lower regions of five different locations 
in the piles to achieve a good representation of the whole composting pile. Samples from CK-1 group and OST group were collected at the days of $0,2,5,8,10,13,15,18,22,26,30$, and 35 , while additional two samples at day 42 and 49 were collected for groups CK-2 and TCS. Each sample was separated into two subsamples where one subsample was tested for physicochemical analysis immediately after sampling. The other part of them was stored as fresh sample at $-20^{\circ} \mathrm{C}$ for later total genomic DNA extraction [15].

Table 1. Experiment Design of Compost in Different Treatment

\begin{tabular}{ll}
\hline Group name $^{*}$ & \multicolumn{1}{c}{ Treatment } \\
CK-1 & $\begin{array}{l}\text { Swine manure + corn straw + without inoculation } \\
\text { CK-2 }\end{array}$ \\
OST & $\begin{array}{l}\text { Swine manure + corn straw + without inoculation } \\
\text { (inoculating at the initial phase of compost) }\end{array}$ \\
TCS & $\begin{array}{l}\text { Swine manure + corn straw + with inoculation } \\
\text { (inoculating at the maturation phase of compost) }\end{array}$ \\
\hline
\end{tabular}

* CK-1, Control group one, following with the composting period of One Step Through (OST) group; CK-2, Control group two, following with the composting period of Two Consecutive Stages (TCS) group; OST, One Step Through group, inoculating $P$. polymyxa at the ratio of $5 \%(\mathrm{v} / \mathrm{w})$ into the composting piles on the initial phase of compost; TCS, Two Consecutive Stages group, inoculating $P$. polymyxa at the ratio of $5 \%(\mathrm{v} / \mathrm{w})$ into the composting piles on the first maturation phase of compost.

\subsection{Index Measurements}

The value of $\mathrm{pH}$ was determined by using a $\mathrm{pH}$ meter (PHS-3E, Shanghai, China) with fresh samples suspended in purified water at 1:10 (w/w) [16]. Electric conductivity (EC) was detected using detecting instrument (DDS-307, Shanghai, China) with fresh samples suspended in sterile water at 1:10 (w/w) [17]. Then it was oscillated 20 minutes followed by filtration, measurement and data recording. Moisture content was measured by weight difference before and after drying the compost samples at $105^{\circ} \mathrm{C}$ for $24 \mathrm{~h}$ [18]. The organic matter (OM) was measured with a muffle furnace at $550^{\circ} \mathrm{C}$ for $8 \mathrm{~h}$ [18]. Water soluble carbon (WSC) was determined by potassium dichromate oxidation in phosphoric acid bath according to the method of Cayuela, Mondini [19], total nitrogen (TN) content was determined by Kjeldahl method described by Castaldi, Garau [20] using Graphite digestion apparatus (SH220N, China) and Kjeldahl apparatus (K9840, China). The ratio of WSC/TN was then calculated. Total phosphorus (TP) was determined according to the molybdenum blue method [21]. The amounts of potassium oxide $\left(\mathrm{K}_{2} \mathrm{O}\right)$ in fertilizers were determined by flame photometric method [22] .The distilled water extract of the composts (1/20 composts/water, w/v) were shaken at $25^{\circ} \mathrm{C}$ for $3.0 \mathrm{~h}$ for the experiment of germination index (GI) which was measured by the method described by Hu, Zheng [23]. The measurement of the number of effective strains in the bio-organic fertilizer produced by two methods OST and TCS was determined according to the method by Herigstad, Hamilton [24].

\subsection{Microbial Diversity}

Genomic DNA was extracted from the composting samples using
Soil DNA Kit (Omega Bio-Tek, Inc., GA, USA) following the manufacturer's protocol, and the extracts were purified using DNA Purifi cation Kit (Tiangen DNA gel extraction kit, China). The DNA extraction was conducted three times for each sample and the DNA extracts were combined before sequencing. The content and quality of extracted DNA were checked by spectrophotometric analysis with NanoDrop ND-2000 (Thermo Fisher Scientific, Wilmington, USA) and on 1\% agarose gel. $16 \mathrm{~S}$ rRNA genes of V3, V4 and V5 hyper variable regions were amplified using the special primers (V3 and V4 regions for forward primers $5{ }^{`}$-CCTACGGRRBGCAS CAGKVRVGAAT-3 ` and reverse primers5`-GGACTACNVGGGT WTCTAATCC-3`, V4 and V5 regions for forward primers 5 '-GTGYCAGCMGCCG CGGTAA-3` and reverse primers 5 `-CTTGTGCGGKCCC CCGYCAATTC-3`). The PCR reaction was performed in $50 \mu \mathrm{L}$ mixture containing $25 \mu \mathrm{L}$ of $2 \times$ Premix Taq (Takara Biotechnology, Dalian Co. Ltd., China), $1 \mu \mathrm{L}$ each of forward and reverse primer (Carlsbad, CA, USA), $3 \mu \mathrm{L}$ of template DNA (20 ng $\mu \mathrm{L}^{-1}$ ) and $20 \mu \mathrm{L}$ of Nuclease-free water. The PCR thermal cycling conditions were programed as initial denaturation $\left(94^{\circ} \mathrm{C}\right.$ for $2 \mathrm{~min}), 32$ cycles of denaturation $\left(94^{\circ} \mathrm{C}\right.$ for $15 \mathrm{~s}$ ), annealing $\left(55^{\circ} \mathrm{C}\right.$ for $\left.30 \mathrm{~s}\right)$, extension $\left(72^{\circ} \mathrm{C}\right.$ for $\left.30 \mathrm{~s}\right)$, and a final extension $\left(72^{\circ} \mathrm{C}\right.$ for $\left.5 \mathrm{~min}\right)$. Paired-end reads were merged using FLASH, and then reads were assigned to each sample according to the unique barcode. Raw reads were trimmed using Quantitative Insights Into Microbial Ecology software package (QIIME, version 1.8.0, http://bio.cug.edu.cn/ qiime/). The qualified sequences were clustered into operational taxonomic units (OTUs) at 97\% sequence identity using UPARSE pipeline embedded in QIIME [25]. Representative sequences from each OTU were assigned taxonomically by using Ribosomal Database Project (RDP) pipeline (http://pyro.cme.msu.edu) at $97 \%$ sequence identity [26]. The alpha-diversity (Simpson, Chao 1, Shannon, ACE) were performed by QIIME software package as described previously [27].

\subsection{Preparation for Pot Experiments}

Chinese Pakchoi seeds and the plant soil were purchased from Flower Market of Dalian Xijiao Gardens, China. The plant soil had the following properties: $\mathrm{pH}$ 6.8, organic matter $372 \mathrm{~g} / \mathrm{kg}$, available N $78.0 \mathrm{mg} / \mathrm{kg}$, available P $130 \mathrm{mg} / \mathrm{kg}$, and available K 41.0 $\mathrm{mg} / \mathrm{kg}$. The plant soil was considered healthy for the growth of Chinese Pakchoi due to no pathogenic bacteria or soil-borne diseases were detected in the soil. The experiment soil used in the disease resistance experiment of Chinese Pakchoi anthracnose was prepared by mixing the healthy soil with $C$. higginsianum $\left(1 \times 10^{6} \mathrm{CFUs} / \mathrm{mL}\right)$ at the rate of $0.5 \%$ dry weight of soil. The growth experiment of Chinese Pakchoi and the disease resistance experiment of Chinese Pakchoi anthracnose were conducted in a greenhouse $\left(25 \pm 2^{\circ} \mathrm{C}\right.$, $70 \%$ Relative Humidity, RH, 10-12h day-light) with $2 \%$ (w/w) loading of the fertilizers [28]. When Chinese Pakchoi seedlings was at 5-leaf stage, they were separated and transplanted from the healthy soil to the pots for further 35 days experiment. Chinese Pakchoi seedlings with 5 leafs were divided into two parts, one for the determination of growth indicators, and the other for the determination of disease resistance indicators. Each of Chinese Pakchoi seedlings were cultivated in pots $(20 \times 20 \times 25 \mathrm{~cm})$ containing $900 \mathrm{~g}$ of soil. Seven pots were contained in each treatment. 


\subsection{Disease Resistance Index}

The disease incidence (DI) was used as an indicator when plants were infected with diseases [29]. The experiment design of DI was shown in Table 2. The calculations of DI and disease resistance index (DRI) [29] were shown in Eq. (1) and (2), respectively, as below:

$$
\begin{gathered}
\mathrm{DI}=(\text { Number of diseased plants }) / \\
\text { (total number of plants }) \times 100 \% \\
\mathrm{DRI}=\left[\left(\mathrm{CK}_{\mathrm{DI}}-\mathrm{T}_{\mathrm{DI}}\right) / \mathrm{CK}_{\mathrm{DI}}\right] \times 100 \%
\end{gathered}
$$

where $\mathrm{CK}_{\mathrm{DI}}$ is the disease incidence of the control treatment and $\mathrm{T}_{\mathrm{DI}}$ is the disease incidence of the treatments. Chemical fertilizer (NPK compound fertilizer) used in this experiment was purchased from Flower Market of Dalian Xijiao Gardens, China.

Table 2. Pot Experiment Design of DI on the Growth of Chinese Pakchoi

\begin{tabular}{lc}
\hline Group name $^{\text {a }}$ & Treatment \\
CK-p1 & Experiment soil \\
CF-p1 & Experiment soil + Chemical Fertilizer \\
OST-p1 & Experiment soil + \\
& Bio-organic Fertilizer produced by OST \\
TCS-p1 & Experiment soil + \\
\hline
\end{tabular}

${ }^{a}$ CK-p1, Control group, loading without any fertilizer;

CF-p1, Chemical Fertilizer group, loading with chemical fertilizer; OST-p1, One Step Through p1 group, loading with bio-organic fertilizer by the method of OST; TCS-p1, Two Consecutive Stages group p1 group, loading with bio-organic fertilizer by the method of TCS.

${ }^{\mathrm{b}}$ Experiment soil, inoculating with $C$. higginsianum $\left(1 \times 10^{6} \mathrm{cfu} / \mathrm{mL}\right)$ at the rate of $0.5 \%$ dry weight of soil into the plant soil.

${ }^{\mathrm{c}}$ All the trial groups loaded the fertilizer with $2 \%(\mathrm{w} / \mathrm{w})$.

\subsection{Physicochemical Parameters of Chinese Pakchoi}

The fertility of bio-organic fertilizers produced by OST and TSC methods was tested by the pot experiments in a greenhouse where the growth of Chinese Pakchoi was monitored (Table 3). Thirty-five days after transplantation, seven Chinese Pakchoi plants in each treatment were randomly sampled for measurements of plant height, root length, leaf length, leaf width, fresh weight and dry weight [9]. The measurements were repeated three times and the average of the measured values was determined as the growth index of each plant. The pigment in chloroplast of Chinese Pakchoi leaves was determined by ethanol extraction spectrophotometry. The soluble reducing sugar was determined by anthrone colorimetry and the soluble protein was determined by Co-omassie Brilliant Blue G-250 staining. The content of vitamin C (Vc) was determined by 2, 6 - two chlorophenol indophenol titration [30]. The content of N, P, K was determined by the method of Gao [30]. Nitrate reductase (NR) was tested using nitrate reductase kit (Solarbio-BC0080, Beijing Solarbio Science \& Technology

\begin{tabular}{|c|c|}
\hline Group name $^{a}$ & Treatment \\
\hline CK-p2 & Healthy soil ${ }^{\mathrm{b}}$ \\
\hline CF-p2 & Healthy soil + Chemical Fertilizer ${ }^{\mathrm{c}}$ \\
\hline OST-p2 & $\begin{array}{ll} & \text { Healthy soil }+ \\
\text { Bio-organic } & \text { Fertilizer produced by OST }\end{array}$ \\
\hline TCS-p2 & $\begin{array}{l}\text { Healthy soil }+ \\
\text { Bio-organic Fertilizer produced by TCS }\end{array}$ \\
\hline $\begin{array}{l}\text { a CK-p2, Contrc } \\
\text { OST-p2, One } \\
\text { fertilizer by } t \\
\text { TCS-p2, Two } \\
\text { bio-organic fe } \\
\text { b Healthy soil, }\end{array}$ & $\begin{array}{l}\text { group; CF-p2, Chemical Fertilizer group; } \\
\text { ep Through p2 group, loading with bio-organic } \\
\text { method of OST; } \\
\text { onsecutive Stages group p2 group, loading with } \\
\text { ilizer by the method of TCS. } \\
\text { oculating without any thing. }\end{array}$ \\
\hline
\end{tabular}
Co., Ltd.).
Table 3. Pot Experiment Design of the Growth of Chinese Pakchoi

\subsection{Statistical Analyses}

Statistical analyses were performed using GraphPad Prism 7.0 for windows. All values were expressed as their mean \pm standard deviations of three replicates, and levels of significance were evaluated using one-way ANOVA with Duncan's multiple range tests. Differences were considered at a significant threshold of $p<0.05$ level.

\section{Conclusions}

\subsection{Physicochemical Parameters of Compost}

The profile of composting parameters such as temperature, moisture content, $\mathrm{pH}, \mathrm{EC}$ and the ratio of WSC/TN from initial phase to first mature phase had a similar trend with each other in the four trial treatments (Fig. S1). On the third day of composting, the average temperature of all the composting piles (Fig. S1(a)) exceeded $50^{\circ} \mathrm{C}$ and lasted for $9 \mathrm{~d}$ which met the basic standards of USEPA (United States Environmental Protection Agency) and USCC (United States Composting Council). The temperature profile showed that group OST entered the thermophilic phase two days earlier than TCS group. Besides, the duration with the average temperature above $55^{\circ} \mathrm{C}$ is $6 \mathrm{~d}$ in OST group while $5 \mathrm{~d}$ in TCS group. Both of these two groups met the standards of Hygienic requirements for harmless disposal of night soil (GB 7959-2012) and Microbial organic fertilizers of China (NY884-2012). The temperature of group TCS increased gradually with the highest temperature of $39.8^{\circ} \mathrm{C}$ achieved on the thermophilic phase of the second stage of compost. The value of moisture content was $27.01 \%$ in group OST, higher than $21.52 \%$ in group TCS (Fig. S1(b)). The value of $\mathrm{pH}$ was 7.85 in group OST, similar as 7.79 in group TCS (Fig. S1(c)). The value of EC was $2.38 \mathrm{mS} / \mathrm{cm}$ in group OST, lower than $2.53 \mathrm{mS} / \mathrm{cm}$ in group TCS (Fig. S1(d)). The ratio of WSC/TN was 23.54 in group OST, higher than 20.64 in group TCS (Fig. $\mathrm{S} 1(\mathrm{e})$ ). However, the difference in these parameters (moisture content, $\mathrm{pH}, \mathrm{EC}$ and WSC/TN) between group OST and group TCS was not significant. In addition, the selected samples from the four treatments at the end of composting also showed no significant 
Table 4. Selected Characters of the Final Results of Composts

\begin{tabular}{|c|c|c|c|c|}
\hline Treatment* & OM (\%) & TN (\%) & TP (\%) & $\mathrm{K}_{2} \mathrm{O}(\%)$ \\
\hline CK-1 & $39.82 \pm 1.86^{\mathrm{a}}$ & $3.10 \pm 0.63^{\mathrm{a}}$ & $3.35 \pm 0.08^{\mathrm{a}}$ & $0.87 \pm 0.13^{\mathrm{a}}$ \\
\hline CK-2 & $38.69 \pm 1.58^{\mathrm{a}}$ & $3.09 \pm 0.26^{\mathrm{a}}$ & $3.42 \pm 0.11^{\mathrm{a}}$ & $0.83 \pm 0.11^{\mathrm{a}}$ \\
\hline OST & $42.38 \pm 1.74^{\mathrm{a}}$ & $3.21 \pm 0.33^{\mathrm{a}}$ & $3.52 \pm 0.18^{\mathrm{a}}$ & $0.89 \pm 0.08^{\mathrm{a}}$ \\
\hline TCS & $40.54 \pm 2.01^{\mathrm{a}}$ & $3.16 \pm 0.11^{\mathrm{a}}$ & $3.47 \pm 0.32^{\mathrm{a}}$ & $0.91 \pm 0.11^{\mathrm{a}}$ \\
\hline
\end{tabular}

Values are expressed as the mean \pm standard deviations. OM: organic matter. TN: total nitrogen. TP, total phosphorus. $\mathrm{K}_{2} \mathrm{O}$, potassium oxide. Same letters in each column represent no significant differences among treatments according to Duncan's multiple range test at thereshold of $p<0.05$ level.

* CK-1, Control group one, following with the composting period of One Step Through (OST) group; CK-2, Control group two, following with the composting period of Two Consecutive Stages (TCS) group; OST, One Step Through group, inoculating $P$. polymyxa at the ratio of $5 \%(\mathrm{v} / \mathrm{w})$ into the composting piles on the initial phase of compost; TCS, Two Consecutive Stages group, inoculating $P$. polymyxa at the ratio of $5 \%(\mathrm{v} / \mathrm{w})$ into the composting piles on the first maturation phase of compost.

difference regarding indexes of GI (Fig. S1(f)), where the value of GI was $103.67 \%$ in group OST and $104 \%$ in group TCS. The other characteristics of final results of composts by the two methods for producing bio-organic fertilizers were shown in Table 4. The difference of parameters $\mathrm{OM}, \mathrm{TN}$, TP, and $\mathrm{K}_{2} \mathrm{O}$ between group OST and group TCS were also not significant. The amount of $P$. polymyxa in bio-organic fertilizers produced by two kinds of methods of OST and TCS was $1 \times 10^{8}$ CFUs and $1 \times 10^{9}$ CFUs, respectively.

Temperature has been considered as one of the most critical parameter in determining composting efficiency. Moreover, it has a close relationship with maturity and disposal harmless [31-33]. The temperature of group OST increased faster than other groups on the initial phase, which could be mainly due to the microbial activity from the inoculated microbial agents as also being reported in previous studies [15, 34]. The temperature profile of group TCS showed that there was a thermophilic phase on the second stage of compost with the main reason being inoculation of biological control agents into the composting piles after the first stage of composting. Moreover, the rapid increase in moisture content could stimulate growth and metabolisms of some microorganisms. In addition, $P$. polymyxa could also hold the capability of metabolizing and degrading organic matters in the composting piles, which was consistent with the traditional process of producing bio-organic fertilizer [3, 9]. The value of EC and GI were also an important parameters in determining the compost stability and maturity [35]. In this study, the results of EC in group OST and group TCS were consistent with the previous study [36]. The end products of the compost via two kinds of method OST and TCS had an positive effect on plants mainly due to that the EC value was below 4.0 $\mathrm{mS} / \mathrm{cm}$ [37]. The indexes of GI in group OST and group TCS (exceeding 100\% in this study) was absent of phytotoxins when
GI was greater than $80 \%$ [38], which was consistent with results reported in the previous study [39]. Overall, combining these above non-differentiated indicators, the end products of compost by the two kinds of methods were consistent with each other in terms of maturity. In additional, it was more important that both group OST and group TCS had a similar concentration of $P$. polymyxa in their corresponding bio-organic fertilizers. The number of microbes in the produced fertilizers met the standard of microbial organic fertilizers of China (NY 884-2012), which indicated that the bio-organic fertilizer produced by these two methods had similar stability. The similar amount of microbes achieved from the two different methods could be due to the sporulation that protected the strains from sterilization during high temperature. Once the temperature reduced to be suitable for spore propagation, the strains revived and the amount of $P$. polymyxa was restored nearly to its initial state [40, 41]. According to the comprehensive evaluation of the above indicators, although there was no significant difference in maturity and stability of compost between OST group and TCS group, it took two weeks less for OST group to complete the production of bio-organic fertilizer than TCS group.

\subsection{Microbial Diversity}

The four indexes of diversity (Chao1, ACE, Shannon and Simpson) reflect the proportion of special microorganisms in rhizosphere soil microorganisms. The results of microbial diversity showed the relative abundance of the top 20 microorganisms at the end of composting (Fig. 1). The value of Chao1 and ACE in group OST showed no significant difference comparing to group TCS (Fig. 1(a) and (c)). There was also no significant difference between group OST and group TCS in terms of two reference indicators, Simpson and Shannon (Fig. 1(b) and (d)). It indicated that group OST had the same species richness and evenness as group TCS. The proportion of $P$. polymyxa in phylum of group TCS was significantly higher than that of group CK-1 and CK-2, however, there was no significant difference compared with that of group OST (Fig. 2). Besides, although group OST was lower in terms of the proportion of $P$. polymyxa in family and genus than that of group TCS by $0.13 \%$ and $0.16 \%$, respectively, the difference was not significant.

In the relative abundance diversity of top 20 microorganisms, group TCS showed higher OTUs than the other trial groups for the main reason that $P$. polymyxa was inoculated into the composting piles on the first maturation phase during the first stage of compost, where the reproduction and distribution of microorganisms were more influenced than that of group OST. The relative abundance of $P$. polymyxa in group OST was lower than in group TCS which could be mainly due to another reason that the proportion of $P$. polymyxa decreased at the high temperature that was beyond the survival limit of $P$. polymyxa. However, the sporulation of $P$. polymyxa was formed in the case of this external stress during the thermophilic phase of composting. As the environmental stress factors decreased, the spores of $P$. polymyxa reproduced. It could result in the number of the strains being maintained at a certain level at the end of composting [41]. The indexes of Shannon and Simpson reflected that the species richness and evenness of compost were affected by the inoculation of antagonistic bacteria [42]. In 

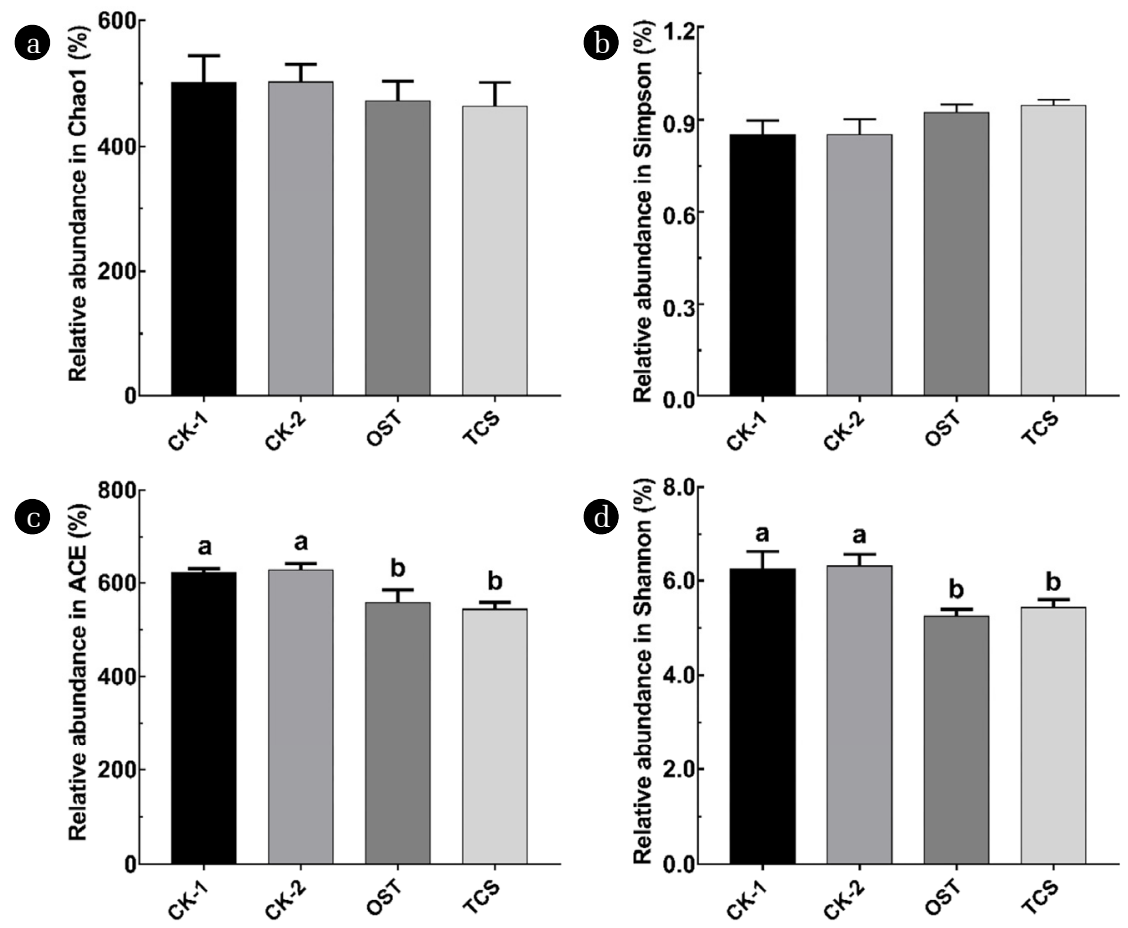

Fig. 1. Chao1, ACE, Simpson and Shannon indexes of the end of composts in different treatments $(p<0.05)$. (a) Chao1; (b) Simpson; (C) ACE; (D) Shannon. CK-1, Control group one, following with the composting period of One Step Through (OST) group; CK-2, Control group two, following with the composting period of Two Consecutive Stages (TCS) group; OST, One Step Through group, inoculating P. polymyxa at the ratio of $5 \%(\mathrm{v} / \mathrm{w})$ into the composting piles on the initial phase of compost; TCS, Two Consecutive Stages group, inoculating $P$. polymyxa at the ratio of $5 \%(\mathrm{v} / \mathrm{w})$ into the composting piles on the first maturation phase of compost.

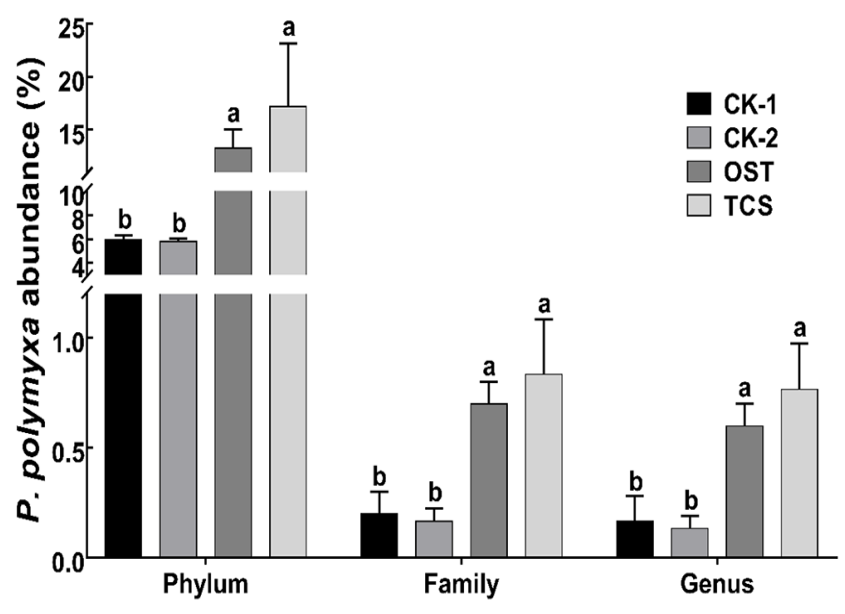

Fig. 2. The relative abundance of $P$. polymyxa OTUs in phylum, family and genus at the end of compost $(p<0.05)$. CK-1, Control group one, following with the composting period of One Step Through (OST) group; CK-2, Control group two, following with the composting period of Two Consecutive Stages (TCS) group; OST, One Step Through group, inoculating $P$. polymyxa at the ratio of $5 \%(\mathrm{v} / \mathrm{w})$ into the composting piles on the initial phase of compost; TCS, Two Consecutive Stages group, inoculating P. polymyxa at the ratio of $5 \%(\mathrm{v} / \mathrm{w})$ into the composting piles on the first maturation phase of compost. this study, the richness and evenness of the antagonistic strain in group OST as well as group TCS were better than that of the control group, which could be attributed to the three factors: 1 ) $P$. polymyxa was one of the dominant species in the composting piles due to the inoculation; 2) $P$. polymyxa had a stronger adaptability than other strains of the composting piles; 3) $P$. polymyxa had the mechanism of forming spores for resisting thermophilic phase of compost. It could be one of the above three factors that the final products of compost, bio-organic fertilizer produced by the novel OST method contained $P$. polymyxa at a certain level consistent with that by the method of TCS. The data of relative abundance of $P$. polymyxa illustrated that the novel method of producing bio-organic fertilizer by OST was feasible in maintaining the stability of the number of strains.

\subsection{Disease Incidence and Disease Resistance Index}

The disease incidence of group CK-p1 and group CF-p1 was 82.46\% and 66.67\%, respectively (Fig. 3). Compared with group CK-p1, the disease incidence of group OST-p1 and group TCS-p1 was reduced by $61.40 \%$ and $68.42 \%$, respectively, in which it was 21.05 $\%$ in group OST-p1 and $14.06 \%$ in group TCS-p1. Disease incidence in group OST-p1 was $7.01 \%$ higher than that in group TCS-p1, however, the difference was not significant. The result of disease resistance index was $74.47 \%$ in group OST-p1 and $82.98 \%$ in group TCS-p1. However, the $8.51 \%$ difference of disease resistance index between TCS-p1and OST-p1 was not significant. 


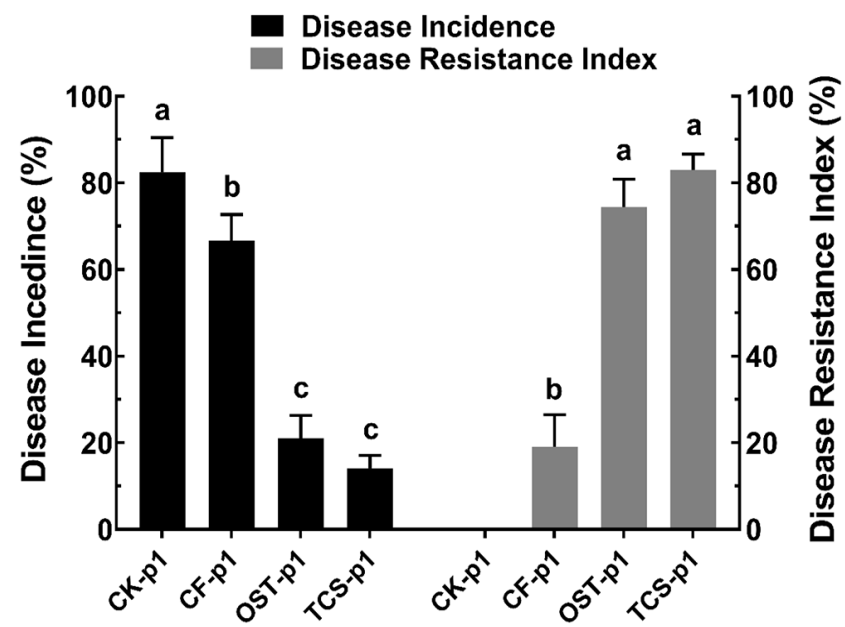

Fig. 3. Disease incidence and disease resistance index of Chinese Pakchoi $(p<0.05)$. CK-p1, Control group, loading without any fertilizer; CF-p1, Chemical Fertilizer group, loading with chemical fertilizer; OST-p1, One Step Through p1 group, loading with bio-organic fertilizer by the method of OST; TCS-p1, Two Consecutive Stages group p1 group, loading with bio-organic fertilizer by the method of TCS. The experiment soil was inoculated with C. higginsianum $\left(1 \times 10^{6} \mathrm{cfu} / \mathrm{mL}\right)$ at the rate of $0.5 \%$ dry weight of soil. All the trial groups loaded the fertilizer with $2 \%(\mathrm{w} / \mathrm{w})$.

C. higginsianum is an ascomycete fungus causing anthracnose disease of many cultivated Brassicaceae [43]. P. polymyxa has the ability to suppress the pathogen of $C$. higginsianum [44]. Therefore, it was used as an antagonistic strain for producing the bio-organic fertilizers as an alternative method of replacing chemical pesticides and insecticides. Disease incidence and disease resistance index were considered as one of the fundamental parameters for evaluating the ability of bio-organic fertilizer to resist the disease pathogen [45]. Bio-organic fertilizer produced by the novel OST method showed no significant difference in disease incidence and disease resistance index with that produced by the traditional TCS method. Although the relative abundance of $P$. polymyxa of group OST was lower than that of group TCS in phylum, family and genus, the number of $P$. polymyxa in group OST was enough to form biofilm on plant roots, which coincided with the colonization sites of pathogen and thereby functioned as a protective layer to prevent access by the pathogens [46]. The results of this study was consistent with previous reports $[47,48]$. Another main reason for no significant difference in the resistance to pathogens by the bio-organic fertilizer produced from OST method and the traditional TCS method was that the spore formed by $P$. polymyxa propagated into bacterial body in the experiment soil. These revived antagonistic strains could inhibit the germination of conidia of the pathogen [49].

\subsection{Fertility of Bio-organic Fertilizer Produced by OST and TCS}

Compared with the control group of CK-p2 (Fig. 4), group OST-p2 increased plant height, root length, leaf length, leaf width, fresh weight and dry weight by 43.14\%, 78.07\%, 45.30\%, 75\%, 154.4\%, $258.4 \%$, respectively. Group OST-p2 reduced the above six growth indicators by $0.14 \%, 7.43 \%, 0.34 \%, 2.05 \%, 3.91 \%$ and $13.07 \%$, respectively, compared to the group TCS-p2. There was no significant difference in the six parameters between group OST-p2 and group TCS-p2 on the growth of Chinese Pakchoi. In addition to the apparent indicators, the intrinsic biochemical indicators also reflected the significant effect of the bio-organic fertilizer produced by the novel OST method on the growth of Chinese Pakchoi. The content of soluble protein, soluble reducing sugar, chlorolphyll and Vitamin C (Fig. S2) in group OST-p2 was $18.31 \mathrm{mg} / \mathrm{g}, 1.37$ $\mathrm{mg} / \mathrm{g}, 0.81 \mathrm{mg} / \mathrm{g}$ and $1.31 \mathrm{mg} / \mathrm{g}$, respectively. Compared with the control group of CK-p2 regarding these four indicators, group OST-p2 increased by $56.56 \%, 89.81 \%, 38.74 \%$ and $46.64 \%$, respectively. The increasing content of these four indicators was $2.76 \%, 2.78 \%, 13.45 \%$, and $6.34 \%$, respectively, less than that for group TCS-p2. However, there was no significant difference in biochemical indicators between group OST-p2 and group TCS-p2. The enzyme activity of nitrate reductase was $7.52 \mathrm{u} / \mathrm{g} \cdot \mathrm{FW}$ in group OST and $8.56 \mathrm{u} / \mathrm{g} \cdot \mathrm{FW}$ in group TCS, which increased by $42.60 \%$ and $62.33 \%$, respectively, compared to the control group of CK-p2. It showed a significant difference in the Nitrate reductase activity between group OST-p2 and group TCS-p2. The content of NPK (Fig. S3) in Chinese Pakchoi showed that the difference between group OST and group TCS was consistent with the different changes of intrinsic biochemical indicators such as soluble protein, compared to the control group of CK-p2.

The fertility of a fertilizer is to promote the growth of crops, vegetables and fruits, which is one of the important part of function of bio-organic fertilizer. Bio-organic fertilizers can not only supply one or more essential macro/micronutrients for plant growth and development, but also provide phytohormones or phytohormones analogues [50]. Another main reason for promoting plant growth was that bio-organic fertilizer could provide or improve the microenvironment for survival of rhizosphere microorganisms [51]. Therefore, in this study, bio-organic fertilizer produced by the novel OST method had enough fertility on the growth of Chinese Pakchoi, which was consistent with the previous report [46].

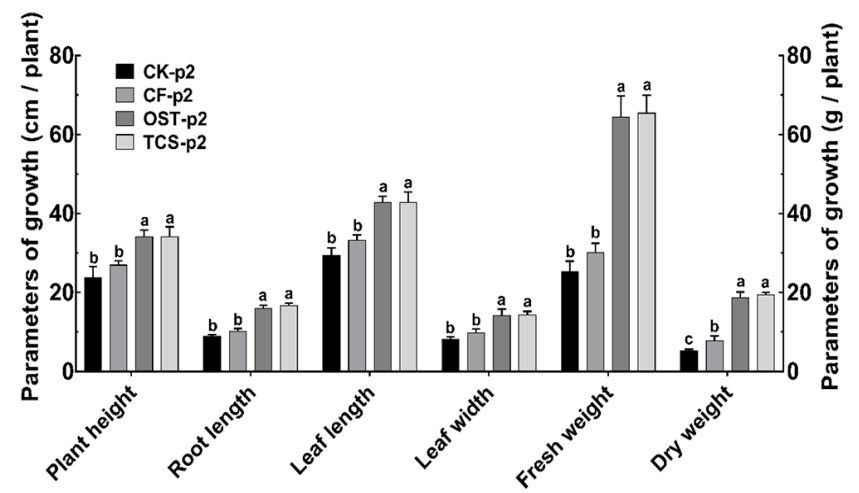

Fig. 4. Parameters of the growth of Chinese Pakchoi $(p<0.05)$. CK-p2, Control group; CF-p2, Chemical Fertilizer group; OST-p2, One Step Through p2 group, loading with bio-organic fertilizer by the method of OST; TCS-p2, Two Consecutive Stages group p2 group, loading with bio-organic fertilizer by the method of TCS. The plant soil contained no disease was considered as healthy soil. All the trial groups loaded the fertilizer with $2 \%(\mathrm{w} / \mathrm{w})$. 
Although the improvement by the rhizosphere microbial communities for plant growth and success cannot be overstated, there were many publications reporting that $P$. polymyxa as a nonpathogenic, probiotic and anti-pathogenic microbes can be used as one of the microbes for commercial compound inoculations [52, 53]. There was no significant difference in the content of $P$. polymyxa in the end product of the composts produced by the two kinds of method, which indicates no significant difference in the fertility of group OST-p2 and group TCS-p2 on the growth of Chinese Pakchoi regarding the common parameters and biochemical parameters such as content of soluble protein, reducing sugars, vitamin $\mathrm{c}$, and chlorophyll. The results were consistent with the findings of previous studies $[54,55]$. The enzyme activity of nitrate reductase in group TCS was higher than that in group OST, which was attributed firstly to the total higher chlorophyll content in TCS group, as was also consistent with previous reports [56]. Secondly, $P$. polymyxa in the bio-organic fertilizer could induce or promote the activity of nitrate reductase of Chinese Pakchoi, which could be one of the mechanisms in resistant disease pathogen, such as C. higginsianum. Furthermore, with the promoting activity of nitrate reductase, the activities of antioxidant enzymes, such as superoxide dismutase, catalase and peroxidase, were significantly altered [57]. This study was coincident with the previous researcher of Singh, Singh [57] that whether it was a disease resistance experiment or a healthy soil planting experiment, the bio-organic fertilizer obtained by the OST method could have the same effect of disease-resisting and growth-promoting as it obtained by the traditional method. In addition, dos Santos, da Silva Ribeiro [58] reported, and was also verified in the present study, that the activity of nitrate reductase was associated with the content of total nitrogen which could be increased with the enhanced activity of nitrate reductase.

The production cost was considered as an important criterial to evaluate the method of producing bio-organic fertilizer. The traditional method requires strict monitoring during the maturity phase of composting to manage timing for inoculating the bio-control microbial agents. Besides, extra efforts for turning and stirring of composting piles are also required due to the relatively long time needed for composting. Therefore, more manpower, material resources and financial cost is inevitable for traditional TCS method. However, the novel OST method for production of bio-organic fertilizer avoided strict monitor for timing of inoculation due to the bio-control microbial agents inoculated at the beginning of composting. The production of bio-organic fertilizer is completed with completion of the composting, which saves time and reduces extra energy and cost investment. Considering the cycle of producing bio-organic fertilizer by the traditional method based on this study, it took about $50 \mathrm{~d}$ to process each batch, and therefore the maximum of 7 batches can be performed each year. However, with the novel OST method, bio-organic fertilizer with the same fertility and quality could be obtained after 35 days of composting for each batch, and therefore, maximum of 10 batches can be achieved each year. Compared with the traditional method, the novel one step through method for producing bio-organic fertilizer improved production efficiency by $42.86 \%$, which was a significant improvement in composting technology.

\section{Conclusions}

Bio-organic fertilizer produced by the novel one step through (OST) method could effectively control the plant diseases and promote the growth of Chinese Pakchoi. Besides, the overall processing time of OST method was 14 days shorter than that of traditional TCS method, although the effect of disease resistant index and grow-promotion in OST method was similar as in TCS method. Overall, there was no significant difference between the two methods for producing bio-organic fertilizer in terms of the disease resistance index or the fertility of bio-organic fertilizer, indicating the reliability of the novel OST method as compared to TCS method. Moreover, the OST method with shorter processing time, less energy and workforce input, could be more economically feasible in terms of production efficiency than TCS method. Taking the year as the production cycle, compared with the traditional method of producing bio-organic fertilizer, the new method can improve the production efficiency by about $40 \%$. Other microorganisms with antibacterial function (utilizing specific antagonistic function for specific application) for use to produce bio-organic fertilizer to control certain plant diseases by the developed OST method worth further research.

\section{Acknowledgment}

This research was financially supported by "13th Five-Year" National Key R\&D Program of China (Grant No. 2016YFD0501406) and the National High Technology Research and Development Program of China (863 Program) (Grant No. 2013AA102805-03).

\section{Author Contributions}

Y.C. (Ph.D. student) conducted all the experiments and wrote the manuscript. X.L. (Dr. Professor) supported funding for the experiments. S.L. (Dr. Professor) supported for experiment field. Y.X. (Dr. Professor) was reviser for manuscript and supervisor for the study.

\section{References}

1. Mahmood I, Imadi SR, Shazadi K, Gul A, Hakeem KR. Effects of pesticides on environment. Plant, soil and microbes: Springer; 2016. p. 253-269.

2. Li R, Tao R, Ling N, Chu G. Chemical, organic and bio-fertilizer management practices effect on soil physicochemical property and antagonistic bacteria abundance of a cotton field: implications for soil biological quality. Soil Till. Res. 2017;167: 30-38.

3. Chen Y, Jiang Z, Wu D, et al. Development of a novel bio-organic fertilizer for the removal of atrazine in soil. J. Environ. Manage. 2019;233:553-560.

4. Ma L, Zhang H-Y, Zhou X-K, et al. Biological control tobacco bacterial wilt and black shank and root colonization by bio-or- 
ganic fertilizer containing bacterium Pseudomonas aeruginosa NXHG29. Appl. Soil Ecol. 2018;129:136-144.

5. Xue C, Shen Z, Hao Y, et al. Fumigation coupled with bio-organic fertilizer for the suppression of watermelon Fusarium wilt disease re-shapes the soil microbiome. Appl. Soil Ecol. 2019;140: 49-56.

6. Larson C. China's lakes of pig manure spawn antibiotic resistance. American Association for the Advancement of Science; 2015.

7. Lim SL, Lee LH, Wu TY. Sustainability of using composting and vermicomposting technologies for organic solid waste biotransformation: recent overview, greenhouse gases emissions and economic analysis. J. Clean. Prod. 2016;111:262-278.

8. Qu C, Chen X, Zhang Z, et al. Long-term effects of bio-organic fertilizer application on soil organic carbon pool and enzyme activity of cucumber continuous cropping. Ying yong sheng tai xue bao. J. Appl. Eecol. 2019;30:3145-3154.

9. Zhang N, Pan R, Shen Y, et al. Development of a novel bio-organic fertilizer for plant growth promotion and suppression of rhizome rot in ginger. Biol. Control. 2017;114:97-105.

10. Yao Y, Zhu F, Hong C, et al. Utilization of gibberellin fermentation residues with swine manure by two-step composting mediated by housefly maggot bioconversion. Waste Manage. 2020;105:339-346.

11. Insam H, De Bertoldi M. Chapter 3 Microbiology of the composting process. In: Diaz L. F., De Bertoldi M., Bidlingmaier W., Stentiford E., editors. Waste Management Series. 8: Elsevier; 2007. p. 25-48.

12. Ren X, Zhang N, Cao M, et al. Biological control of tobacco black shank and colonization of tobacco roots by a Paenibacillus polymyxa strain C5. Biol. Fertil. Soils. 2012;48:613-620.

13. Kim YS, Balaraju K, Jeon Y. Biological control of apple anthracnose by Paenibacillus polymyxa APEC128, an antagonistic rhizobacterium. Plant Pathol. J. 2016;32:251.

14. Padda KP, Puri A, Chanway CP. Paenibacillus polymyxa: A prominent biofertilizer and biocontrol agent for sustainable agriculture. Agriculturally Important Microbes for Sustainable Agriculture: Springer; 2017. p. 165-191.

15. Yu Z, Tang J, Liao H, et al. The distinctive microbial community improves composting efficiency in a full-scale hyperthermophilic composting plant. Bioresour. Technol. 2018;265: 146-154.

16. Zeng G, Yu M, Chen Y, et al. Effects of inoculation with Phanerochaete chrysosporium at various time points on enzyme activities during agricultural waste composting. Bioresour. Technol. 2010;101:222-227.

17. Chikae M, Ikeda R, Kerman K, Morita Y, Tamiya E. Estimation of maturity of compost from food wastes and agro-residues by multiple regression analysis. Bioresour. Technol. 2006;97: 1979-1985.

18. Zeng G, Yu Z, Chen Y, et al. Response of compost maturity and microbial community composition to pentachlorophenol (PCP)-contaminated soil during composting. Bioresour. Technol. 2011;102:5905-5911.

19. Cayuela M L, Mondini C, Insam H, Sinicco T, Franke-Whittle I. Plant and animal wastes composting: Effects of the $\mathrm{N}$ source on process performance. Bioresour. Technol. 2009;100:3097-3106.
20. Castaldi P, Garau G, Melis P. Maturity assessment of compost from municipal solid waste through the study of enzyme activities and water-soluble fractions. Waste Manage. 2008;28: 534-540.

21. Murphy J, Riley JP. A modified single solution method for the determination of phosphate in natural waters. Anal. Chim. Acta. 1962;27:31-36.

22. Karami S. Comparison of Three Potassium Determination Methods in Fertilizers. 2016.

23. Hu W, Zheng G, Fang D, et al. Bioleached sludge composting drastically reducing ammonia volatilization as well as decreasing bulking agent dosage and improving compost quality: A case study. Waste Management. 2015;44:55-62.

24. Herigstad B,Hamilton M, Heersink J. How to optimize the drop plate method for enumerating bacteria. J. Microbiol. Methods. 2001;44:121-129.

25. Edgar RC. Search and clustering orders of magnitude faster than BLAST. Bioinformatics 2010;26:2460-2461.

26. Mcdonald D, Price MN, Goodrich J, et al. An improved Greengenes taxonomy with explicit ranks for ecological and evolutionary analyses of bacteria and archaea. ISME. 2012;6:610.

27. Caporaso JG, Kuczynski J, Stombaugh J, et al. QIIME allows analysis of high-throughput community sequencing data. Nature methods. 2010;7:335.

28. Zhang N, Wu K, He X, et al. A new bioorganic fertilizer can effectively control banana wilt by strong colonization with Bacillus subtilis N11. Plant Soil. 2011;344:87-97.

29. Sebesta J, Zwatz B, Corazza L, Roderick H. Disease resistance index as a multi-site indicator of the effectiveness of plant genotypes against diseases. Ochrana Rostlin-UZPI (Czech Republic). 1995.

30. Gao J. Experimental guidance for plant physiology. China Higher Education Press, Beijing (Chinese). 2006.

31. Hu D, Wang R, Yan J, Xu C, Wang Y. A pilot ecological engineering project for municipal solid waste reduction, disinfection, regeneration and industrialization in Guanghan City, China1Paper presented at ICEE 96 International Conference of Ecological Engineering, Beijing, China, 7-11 October 1996.1. Ecol. Eng. 1998;11:129-138.

32. Sun Q, Chen J, Wei Y, et al. Effect of semi-continuous replacements of compost materials after inoculation on the performance of heat preservation of low temperature composting. Bioresour. Technol. 2019;279:50-56.

33. Vitosh-Sillman S, Loy JD, Brodersen B, et al. Effectiveness of composting as a biosecure disposal method for porcine epidemic diarrhea virus (PEDV)-infected pig carcasses. Porcine health manag. 2017;3:22.

34. Wang Q, Li R, Cai H, et al. Improving pig manure composting efficiency employing Ca-bentonite. Ecol. Eng. 2016;87:157-161.

35. Sellami F, Hachicha S, Chtourou M, Medhioub K, Ammar E. Maturity assessment of composted olive mill wastes using UV spectra and humification parameters. Bioresour. Technol. 2008;99:6900-6907.

36. Storino F, Arizmendiarrieta J, Irigoyen I, Muro J, Aparicio-Tejo PM. Meat waste as feedstock for home composting: Effects on the process and quality of compost. Waste Manag. 2016; 56:53-62. 
37. García C,Hernández T, Costa F. Study on water extract of sewage sludge composts. Soil Sci. Plant Nutr. 1991;37:399-408.

38. Zucconi F, Pera A, Forte M, De Bertoldi M. Evaluating toxicity of immature compost. BioCycle (USA). 1981.

39. Barral MT, Paradelo R. A review on the use of phytotoxicity as a compost quality indicator. Dyn. Soil Dyn. Plant. 2011;5: 36-44.

40. Atkinson A, Eley K, Cripps R. Sporulation-deficient thermophilic microorganisms for the production of ethanol. Google Patents; 2016.

41. Malleck T, Daufouy G, André S, Broussolle V, Planchon S. Temperature impacts the sporulation capacities and spore resistance of Moorella thermoacetica. Food Microbiol. 2018;73: 334-341.

42. Gorelick R. Combining richness and abundance into a single diversity index using matrix analogues of Shannon's and Simpson's indices. Ecography 2006;29:525-530.

43. O'connell R, Herbert C, Sreenivasaprasad S et al. A novel Arabidopsis-Colletotrichum pathosystem for the molecular dissection of plant-fungal interactions. Mol. Plant Microb. Interact. 2004;17:272-282.

44. Rathore R, Germaine KJ, Forristal PD, Spink J, Dowling DN. Meta-omics approach to unravel the endophytic bacterial communities of Brassica napus and other agronomically important crops in response to agricultural practices. Endophytes for a Growing World. 2019;232-249.

45. Acar Ü, Kesbiç OS, Yılmaz S, Gültepe N, Türker A. Evaluation of the effects of essential oil extracted from sweet orange peel (Citrus sinensis) on growth rate of tilapia (Oreochromis mossambicus) and possible disease resistance against Streptococcus iniae. Aquaculture 2015;437:282-286.

46. Timmusk S,Grantcharova N, Wagner EGH. Paenibacillus polymyxa Invades Plant Roots and Forms Biofilms. Appl. Environ. Microbiol. 2005;71:7292-7300.

47. Fira D, Dimkić I, Berić T, Lozo J, Stanković S. Biological control of plant pathogens by Bacillus species. J. Biotechnol. 2018;285:44-55.

48. Chowdhury SP, Dietel K, Rändler M, et al. Effects of Bacillus amyloliquefaciens FZB42 on lettuce growth and health under pathogen pressure and its impact on the rhizosphere bacterial community. PLoS One. 2013;8:e68818.

49. Wang B, Zhou J, Huang Z, et al. Biocontrol efficacy of a Paenibacillus polymyxa strain against black rot of sweet potato and its action mechanism. Acta. Agric. Jiangxi. 2017;29:40-43.

50. Miri A, Gholamalizadeh Ahangar A, Ghorbani M, Shirmohammadi E. The effect of PGPR and alfalfa extract on macronutrient and micronutrient contents of sorghum (Sorghum vulgare). Iran Agric. Res. 2016;35:96-103.

51. Sarabia M, Cazares S, González-Rodríguez A, et al. Plant growth promotion traits of rhizosphere yeasts and their response to soil characteristics and crop cycle in maize agroecosystems. Rhizosphere 2018;6:67-73.

52. Hong CE, Kwon SY, Park JM. Biocontrol activity of Paenibacillus polymyxa AC-1 against Pseudomonas syringae and its interaction with Arabidopsis thaliana. Microbiol. Res. 2016;185: 13-21.

53. Naghmouchi K, Baah J, Cudennec B, Drider D. Required characteristics of Paenibacillus polymyxa JB-0501 as potential probiotic. Arch. Microbiol. 2013;195:537-543.

54. Khalil NH, Agah RJ. Effect of Chemical, Organic and Bio Fertilization on Growth and Yield of Strawberry Plant. Int. J. Adv. Chem. Eng. Biol. Sci. 2017;4:167-171.

55. Zhao J, Liu J, Liang $H$, et al. Manipulation of the rhizosphere microbial community through application of a new bio-organic fertilizer improves watermelon quality and health. PLoS One. 2018;13:e0192967.

56. Vishwakarma D, Thakur J, Singh P. Effect of PGPR consortia on nitrate reductase and chlorophyll content of flag leaf of wheat. IJCS. 2017;5:672-674.

57. Singh N, Singh A, Singh D. Autotoxicity of maize and its mitigation by plant growth promoting rhizobacterium Paenibacillus polymyxa. Allelopathy J. 2010;25:195-204.

58. Dos Santos SG, Da Silva Ribeiro F, Da Fonseca CS, et al. Development and nitrate reductase activity of sugarcane inoculated with five diazotrophic strains. Arch. Microbiol. 2017;199:863-873. 